\title{
A large-sample crisis? Exaggerated false positives by popular differential expression methods
}

\author{
Yumei Li ${ }^{1, \dagger}$, Xinzhou Ge ${ }^{2, \dagger}$, Fanglue Peng ${ }^{3}$, Wei Li ${ }^{1, *}$, Jingyi Jessica Li $^{2,4,5,6,7, *}$
}

${ }^{1}$ Division of Computational Biomedicine, Department of Biological Chemistry, School of

Medicine, University of California, Irvine, Irvine, CA 92697, USA

${ }^{2}$ Department of Statistics, University of California, Los Angeles, CA 90095

${ }^{3}$ Department of Molecular and Cellular Biology, Baylor College of Medicine, Houston, TX

77030, USA

${ }^{4}$ Interdepartmental Program in Bioinformatics, University of California, Los Angeles, CA 90095

${ }^{5}$ Department of Human Genetics, University of California, Los Angeles, CA 90095

${ }^{6}$ Department of Computational Medicine, University of California, Los Angeles, CA 90095

${ }^{7}$ Department of Biostatistics, University of California, Los Angeles, CA 90095

†These authors contributed equally to this work

*Corresponding authors. Email: wei.li@uci.edu and lijy03@g.ucla.edu 


\begin{abstract}
We report a surprising phenomenon about identifying differentially expressed genes (DEGs) from population-level RNA-seq data: two popular bioinformatics methods, DESeq2 and edgeR, have unexpectedly high false discovery rates (FDRs). Via permutation analysis on an immunotherapy RNA-seq dataset, we observed that DESeq2 and edgeR identified even more DEGs after samples' condition labels were randomly permuted. Motivated by this, we evaluated six DEG identification methods (DESeq2, edgeR, limma-voom, NOISeq, dearseq, and the Wilcoxon rank-sum test) on population-level RNA-seq datasets. We found that the FDR control was often failed by the three popular parametric methods - DESeq2, edgeR, and limma-voomand the new non-parametric method dearseq. In particular, the actual FDRs of DESeq2 and edgeR sometimes exceeded $20 \%$ when the target FDR threshold was only $5 \%$. Although NOISeq, a non-parametric method used by GTEx, controlled the FDR better than the other four methods did, its power was much lower than that of the Wilcoxon rank-sum test, a classic nonparametric test that consistently controlled the FDR and achieved good power in our evaluation. Based on these results, for population-level RNA-seq studies, we recommend the Wilcoxon rank-sum test.
\end{abstract}

\title{
Main text
}

RNA-seq is an approach to transcriptome profiling using deep-sequencing technologies ${ }^{1-3}$. Since RNA-seq was developed more than one decade ago, it has become an indispensable tool for genome-wide transcriptomic studies. One primary research task in these studies is the identification of DEGs between two conditions (e.g., tumor and normal samples) ${ }^{2}$. A longstanding, core challenge in this task is the small sample size, which is typically two or three 
replicates per condition. Many statistical methods have been developed to address this issue by making parametric, restrictive distributional assumptions on RNA-seq data, and the two most popular methods of this type are DESeq $2^{4}$ and edgeR ${ }^{5}$. However, as sample sizes have become large in population-level RNA-seq studies, where dozens to thousands of samples were collected from individuals ${ }^{6,7}$, a natural question to ask is whether DESeq2 and edgeR remain appropriate.

To evaluate the performance of DESeq2 and edgeR, we applied both methods to 13 populationlevel RNA-seq datasets with total sample sizes ranging from 100 to 1376 (Supplementary Table 1). We found that DESeq2 and edgeR had large discrepancies in the DEGs they identified on these datasets (Supplementary Fig. 1). In particular, $23.71 \%-75 \%$ of the DEGs identified by DESeq2 were missed by edgeR. The most surprising result is from an immunotherapy dataset (including 51 pre-nivolumab and 58 on-nivolumab anti-PD-1 therapy patients) ${ }^{8}$ : DESeq2 and edgeR had only an $8 \%$ overlap in the DEGs they identified (DESeq2 and edgeR identified 144 and 319 DEGs, respectively, with a union of 427 DEGs but only 36 DEGs in common). This phenomenon raises a critical question: did DESeq2 and edgeR reliably control their false discovery rates (FDRs) to the target $5 \%$ on this dataset?

To answer this question, we generated 668 negative-control datasets by randomly permuting the two-condition labels (pre-nivolumab and on-nivolumab) of the 109 RNA-seq samples in this immunotherapy dataset (Methods). Since any DEGs identified from these permuted datasets are known as false positives, we used these permuted datasets to evaluate the FDRs of DESeq2 and edgeR. Surprisingly, DESeq2 and edgeR identified more DEGs from $84.88 \%$ and $78.89 \%$ of these permuted datasets than from the original dataset (Fig. 1A). In particular, DESeq2 and 
edgeR mistakenly identified 16,791 and 13,448 genes as DEGs, respectively, from at least one permuted dataset (Fig. 1B). Even more, as many as $56.25 \%$ and $89.34 \%$ of the DEGs, which DEseq2 and edgeR identified from the original dataset, were also identified as DEGs from at least one permuted dataset, suggesting that these DEGs were spurious (Fig. 1C). These results raise the caution about exaggerated false positives by DESeq2 and edgeR on the original dataset.

What's more counter-intuitive, the genes with larger fold changes estimated by DESeq2 and edgeR (between the two conditions in the original dataset) were more likely identified as DEGs by the two methods from the permuted datasets (Fig. 1D and Supplementary Fig. 2). As biologists tend to believe that these genes are more likely true DEGs (which is not necessarily true because a dataset may contain no true DEGs at all), the fact that these genes are false positives would waste experimental validation efforts.

Out of curiosity and as a means of verification, we investigated the biological functions of the spurious DEGs identified by DESeq2 or edgeR from the permuted datasets. Unexpectedly, these spurious DEGs were enriched in immune-related gene ontology (GO) terms (Fig. 1E). Hence, if these spurious DEGs were not removed by FDR control, they would mislead researchers to believe that there was an immune response difference between pre-nivolumab and on-nivolumab patients, a surely undesirable consequence that DEG analysis must avoid.

Then a question follows: why did DESeq2 and edgeR make so many false positive discoveries from this immunotherapy dataset? Our immediate hypothetical reason was the violation of the negative binomial model assumed by both DESeq2 and edge ${ }^{9}$. To check this hypothesis, we 
separated all genes into two groups: (1) the genes identified as DEGs from any permuted datasets and (2) all the other genes; then we evaluated how well the negative binomial model fit to the genes in each group. In line with our hypothesis, the model fitting was worse for the genes in the first group, consistent with the fact these genes were spurious DEGs (Fig. 1F and

\section{Supplementary Fig. 3).}

Motivated by these findings, we further benchmarked DESeq2 and edgeR along with four other representative DEG identification methods on this immunotherapy dataset and the other 12 population-level RNA-seq datasets from the Genotype-Tissue Expression (GTEx) project ${ }^{7}$ and the Cancer Genome Atlas (TCGA) ${ }^{6}$ (Supplementary Table 1). The four representative methods include two popular methods limma-voom ${ }^{10,11}$ and NOISeq ${ }^{12}$, a new method dearseq ${ }^{13}$ (which claimed to overcome the FDR control issue of DESeq2 and edgeR on large-sample-size data), and the classic Wilcoxon rank-sum test ${ }^{14}$. Note that DESeq2, edgeR, and limma-voom are parametric methods that assume parametric models for data distribution, while NOISeq, dearseq, and the Wilcoxon rank-sum test are non-parametric methods that are less restrictive but require large sample sizes to have good power. (The GTEx project used DESeq2 and NOISeq for DEG identification.) Using permutation analysis on these datasets, we found that DESeq2 and edgeR consistently showed exaggerated false positives (reflected by their underestimated FDRs) compared to the other four methods (Supplementary Fig. 4-15).

While the permutation analysis created true negatives (non-DEGs) to allow FDR evaluation, it did not allow the evaluation of DEG identification power, which requires true positives (DEGs) to be known. Hence, we generated 50 (identically and independently distributed) semi-synthetic 
datasets with known true DEGs and non-DEGs from each of the 12 GTEx and TCGA datasets.

Then we used these semi-synthetic datasets to evaluate the FDRs and power of the six DEG identification methods (Methods). In the comparison between 386 heart left ventricle samples and 372 atrial appendage samples in a GTEx dataset, only the Wilcoxon rank-sum test consistently controlled the FDR under a range of thresholds from $0.001 \%$ to $5 \%$ (Fig. 2A). In contrast, the other five methods, especially DESeq2 and edgeR, failed to control the FDR consistently. Moreover, we compared the power of the six methods conditional on their actual FDRs (Methods). (Due to the tradeoff between FDR and power, power comparison is only valid when FDR is equal.) As shown in Fig. 2A, the Wilcoxon rank-sum test outperformed the other five methods in terms of power.

Finally, to investigate how sample sizes influence the performance of the six methods, we downsampled each semi-synthetic dataset to obtain per-condition sample sizes ranging from 2 to 100 . Again, only the Wilcoxon rank-sum test consistently controlled the FDR at all sample sizes (Fig. 2B). Granted, at the FDR threshold 1\%, the Wilcoxon rank-sum test had almost no power when the per-condition sample size was smaller than 8 - an expected phenomenon for its nonparametric nature. However, when the per-condition sample size exceeded 8 , the Wilcoxon rank-sum test achieved comparable or better power compared with the three parametric methods (DESeq2, edgeR, and limma-voom) and the new method dearseq, and it clearly outpowered NOIseq (Fig. 2B). These observations were consistent across all 600 semi-synthetic datasets (Supplementary Figs. 16-26). In summary, when the per-condition sample size is less than 8 , parametric methods may be used because their power advantage may outweigh their possibly 
exaggerated false positives; however, for large-sample-size data, the Wilcoxon rank-sum test is the best choice for its solid FDR control and good power.

The three parametric methods_-DESeq2, edgeR, and limma- have long been dominant in transcriptomic studies. For example, the GTEx project, a consortium effort studying gene expression and regulation in normal human tissues, used DESeq 2 coupled with NOISeq to find DEGs between tissues ${ }^{15}$; several studies applied edgeR or limma to TCGA RNA-seq data to find DEGs between tumor and normal samples ${ }^{16-18}$; with increasing attention on the immunotherapy, researchers used DESeq2 to detect DEGs between responders and non-responders of the immunotherapy ${ }^{8,19}$. However, while the three parametric methods were initially designed to address the small-sample-size issue, these population-level studies had much larger sample sizes (at least dozens) and thus no longer needed restrictive parametric assumptions. Moreover, violation of parametric assumptions may lead to ill-behaved P-values and thus failed FDR control $^{20}$, an issue independent of the sample size.

In this study, we showed the superiority of the Wilcoxon rank-sum test, a powerful and robust non-parametric test also known as the Mann-Whitney test developed in the $1940 \mathrm{~s}^{14,21-24}$, for analyzing large-sample-size RNA-seq datasets. The Wilcoxon rank-sum test is known to be especially powerful for skewed distributions ${ }^{25}$, as is the case with gene expression counts measured by RNA-seq. Our results also echo the importance of verifying FDR control by permutation analysis. Beyond RNA-seq data analysis, our study suggests that, for populationlevel studies with large sample sizes, classic non-parametric statistical methods should be considered as the baseline methods for data analysis and new method benchmarking. 
Finally, we note that, unlike DESeq2, edgeR, limma-voom, and dearseq, the Wilcoxon rank-sum test is a non-regression-based method, making it unable to adjust for confounders. Hence, to use the Wilcoxon rank-sum test for DEG identification, researchers must first normalize RNA-seq samples to remove possible confounder effects. Another limitation of the Wilcoxon rank-sum test is that it only applies to two-condition comparisons. To compare more than two conditions on population-level data, we recommend the Kruskal-Wallis test, also known as the one-way ANOVA on ranks ${ }^{26}$.

\section{Acknowledgements}

We thank Jason Sheng Li of Wei Li lab for suggestions on the title. We also thank other members of Wei Li lab and Jingyi Jessica Li lab for helpful discussions.

\section{Funding}

This work was supported by the following grants: The U.S. National Institutes of Health R01CA193466 and R01CA228140 (to W.L.); NIH/NIGMS R01GM120507 and R35GM140888, NSF DBI-1846216 and DMS-2113754, Johnson \& Johnson WiSTEM2D Award, Sloan Research Fellowship, and UCLA David Geffen School of Medicine W.M. Keck Foundation Junior Faculty Award (to J.J.L.).

\section{Author contributions}

Y.L., W.L. and J.J.L. conceived and supervised this project. Y.L. and X.Z. performed the data analysis. Y.L., X.Z., F.P., J.J.L and W.L. interpreted the data and wrote the manuscript.

\section{Competing interests}

The authors declare no competing financial interests. 


\section{Data availability}

All the permuted and semi-synthetic datasets used to generated results can be found at Zenodo via https://doi.org/10.5281/zenodo.5241320.

\section{Code availability}

All the codes used to generate results can be found at GitHub via URL

https://github.com/xihuimeijing/DEGs_Analysis_FDR.

A tutorial for identifying DEGs using the Wilcoxon rank-sum test can be found at

https://rpubs.com/LiYumei/806213.

\section{Supplementary Materials}

Materials and Methods

Supplementary Figs. 1 to 26

Supplementary Table 1

\section{References}

$1 \quad$ Nagalakshmi, U. et al. The transcriptional landscape of the yeast genome defined by RNA sequencing. Science 320, 1344-1349, doi:10.1126/science.1158441 (2008).

2 Stark, R., Grzelak, M. \& Hadfield, J. RNA sequencing: the teenage years. Nat Rev Genet 20, 631-656, doi:10.1038/s41576-019-0150-2 (2019).

3 Wang, Z., Gerstein, M. \& Snyder, M. RNA-Seq: a revolutionary tool for transcriptomics. Nat Rev Genet 10, 57-63, doi:10.1038/nrg2484 (2009).

4 Love, M. I., Huber, W. \& Anders, S. Moderated estimation of fold change and dispersion for RNA-seq data with DESeq2. Genome Biol 15, 550, doi:10.1186/s13059-014-0550-8 (2014).

5 Robinson, M. D., McCarthy, D. J. \& Smyth, G. K. edgeR: a Bioconductor package for differential expression analysis of digital gene expression data. Bioinformatics 26, 139-140, doi:10.1093/bioinformatics/btp616 (2010).

6 Cancer Genome Atlas Research, N. et al. The Cancer Genome Atlas Pan-Cancer analysis project. Nat Genet 45, 1113-1120, doi:10.1038/ng.2764 (2013).

7 Consortium, G. T. The GTEx Consortium atlas of genetic regulatory effects across human tissues. Science 369, 1318-1330, doi:10.1126/science.aaz1776 (2020).

8 Riaz, N. et al. Tumor and Microenvironment Evolution during Immunotherapy with Nivolumab. Cell 171, 934-949 e916, doi:10.1016/j.cell.2017.09.028 (2017).

9 Hawinkel, S., Rayner, J. C. W., Bijnens, L. \& Thas, O. Sequence count data are poorly fit by the negative binomial distribution. PLoS One 15, e0224909, doi:10.1371/journal.pone.0224909 (2020). 
Ritchie, M. E. et al. limma powers differential expression analyses for RNA-sequencing and microarray studies. Nucleic Acids Res 43, e47, doi:10.1093/nar/gkv007 (2015).

11 Law, C. W., Chen, Y., Shi, W. \& Smyth, G. K. voom: Precision weights unlock linear model analysis tools for RNA-seq read counts. Genome Biol 15, R29, doi:10.1186/gb-2014-15-2-r29 (2014).

12 Tarazona, S. et al. Data quality aware analysis of differential expression in RNA-seq with NOISeq R/Bioc package. Nucleic Acids Res 43, e140, doi:10.1093/nar/gkv711 (2015).

13 Gauthier, M., Agniel, D., Thiebaut, R. \& Hejblum, B. P. dearseq: a variance component score test for RNAseq differential analysis that effectively controls the false discovery rate. NAR Genom Bioinform $\mathbf{2}$, lqaa093, doi:10.1093/nargab/lqaa093 (2020).

14 Wilcoxon, F. Individual comparisons of grouped data by ranking methods. J Econ Entomol 39, 269, doi:10.1093/jee/39.2.269 (1946).

15 Mele, M. et al. The human transcriptome across tissues and individuals. Science 348, 660-665, doi:10.1126/science.aaa0355 (2015).

Peng, L. et al. Large-scale RNA-Seq Transcriptome Analysis of 4043 Cancers and 548 Normal Tissue Controls across 12 TCGA Cancer Types. Sci Rep 5, 13413, doi:10.1038/srep13413 (2015).

17 Rosario, S. R. et al. Pan-cancer analysis of transcriptional metabolic dysregulation using The Cancer Genome Atlas. Nat Commun 9, doi:10.1038/s41467-018-07232-8 (2018).

18 Tang, Z. et al. GEPIA: a web server for cancer and normal gene expression profiling and interactive analyses. Nucleic Acids Res 45, W98-W102, doi:10.1093/nar/gkx247 (2017).

19 Gide, T. N. et al. Distinct Immune Cell Populations Define Response to Anti-PD-1 Monotherapy and AntiPD-1/Anti-CTLA-4 Combined Therapy. Cancer Cell 35, 238-255 e236, doi:10.1016/j.ccell.2019.01.003 (2019). Multiple Testing. J R Stat Soc B 57, 289-300, doi:10.1111/j.2517-6161.1995.tb02031.x (1995). Mann, H. B. \& Whitney, D. R. On a Test of Whether one of Two Random Variables is Stochastically Larger than the Other. Annals of Mathematical Statistics 18, 50-60, doi:10.1214/aoms/1177730491 (1947). J. L. Hodges, J. \& Lehmann, E. L. The Efficiency of Some Nonparametric Competitors of the t-Test. The Annals of Mathematical Statistics 27, 324-335 (1956). Chernoff, H. \& Savage, I. R. Asymptotic Normality and Efficiency of Certain Nonparametric Test Statistics. Ann. Math. Statist 29, 972-994, doi:10.1214/aoms/1177706436 (1958). Fay, M. P. \& Proschan, M. A. Wilcoxon-Mann-Whitney or t-test? On assumptions for hypothesis tests and multiple interpretations of decision rules. Stat Surv 4, 1-39, doi:10.1214/09-SS051 (2010). Krzywinski, M. \& Altman, N. Nonparametric tests. Nature Methods 11, 467-468, doi:10.1038/nmeth.2937 (2014). 


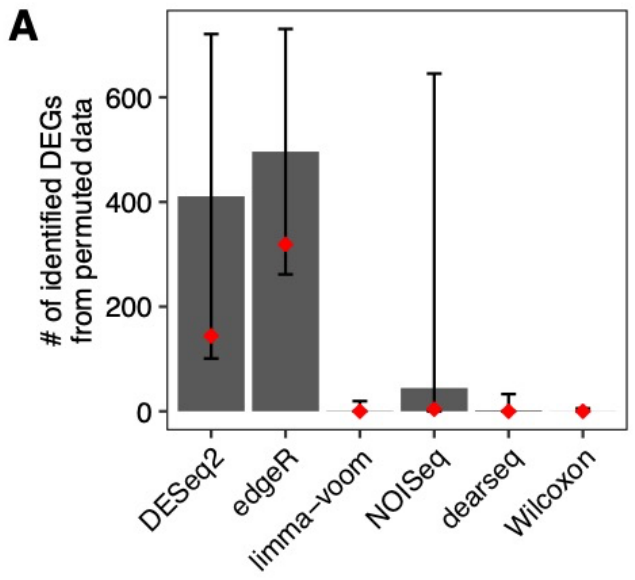

- \# of identified DEGs from the original data

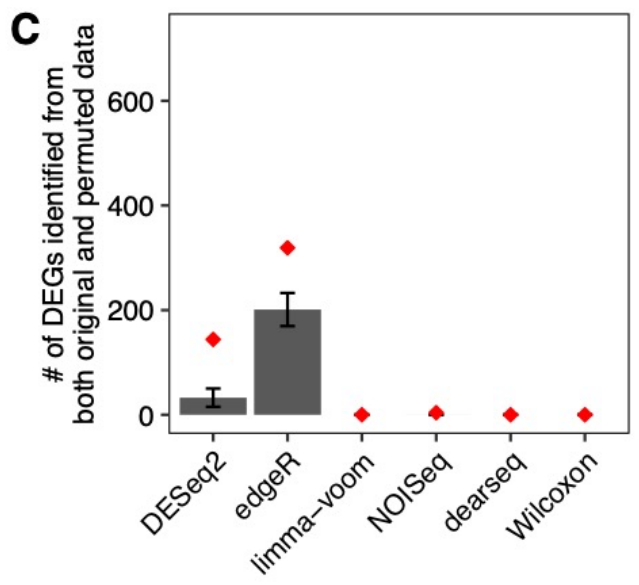

• \# of identified DEGs from the original data

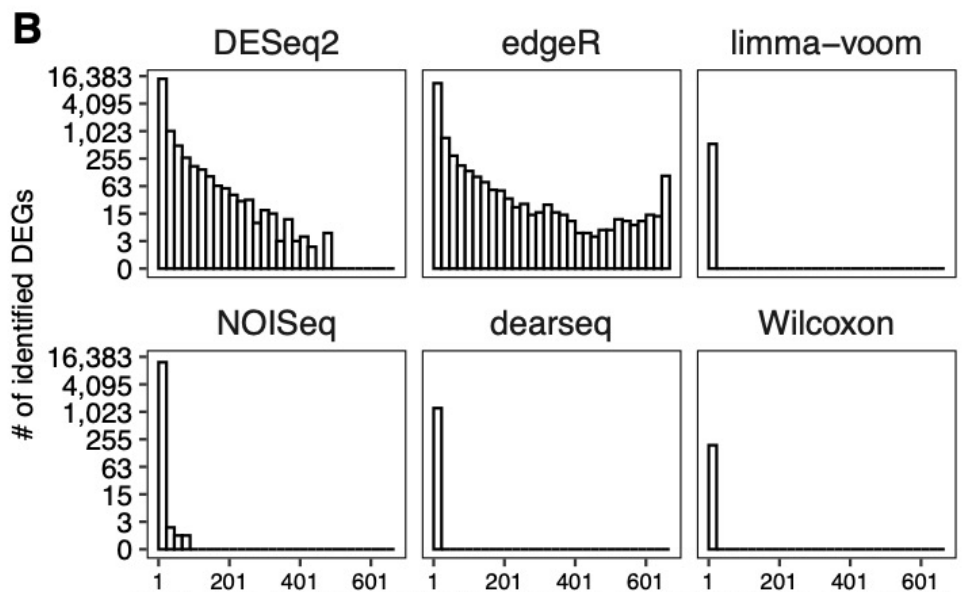

$(0.1 \%)(30 \%)(60 \%)(90 \%)(0.1 \%)(30 \%)(60 \%)(90 \%)(0.1 \%)(30 \%)(60 \%)(90 \%)$

\# of permuted datasets where a gene is wrongly identified as a DEG

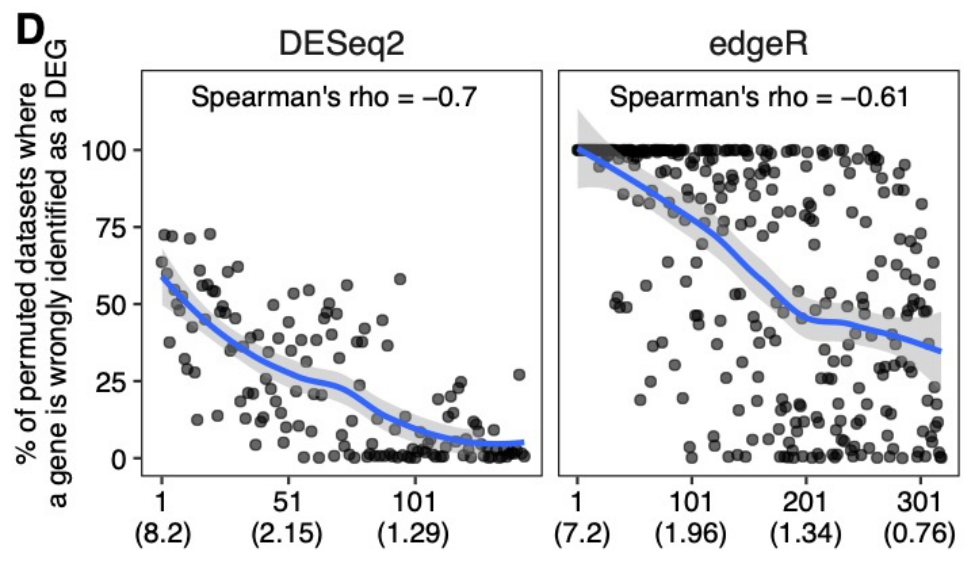

Rank of abs. log2(fold-change)

(from high to low) of DEGs from the original data
E
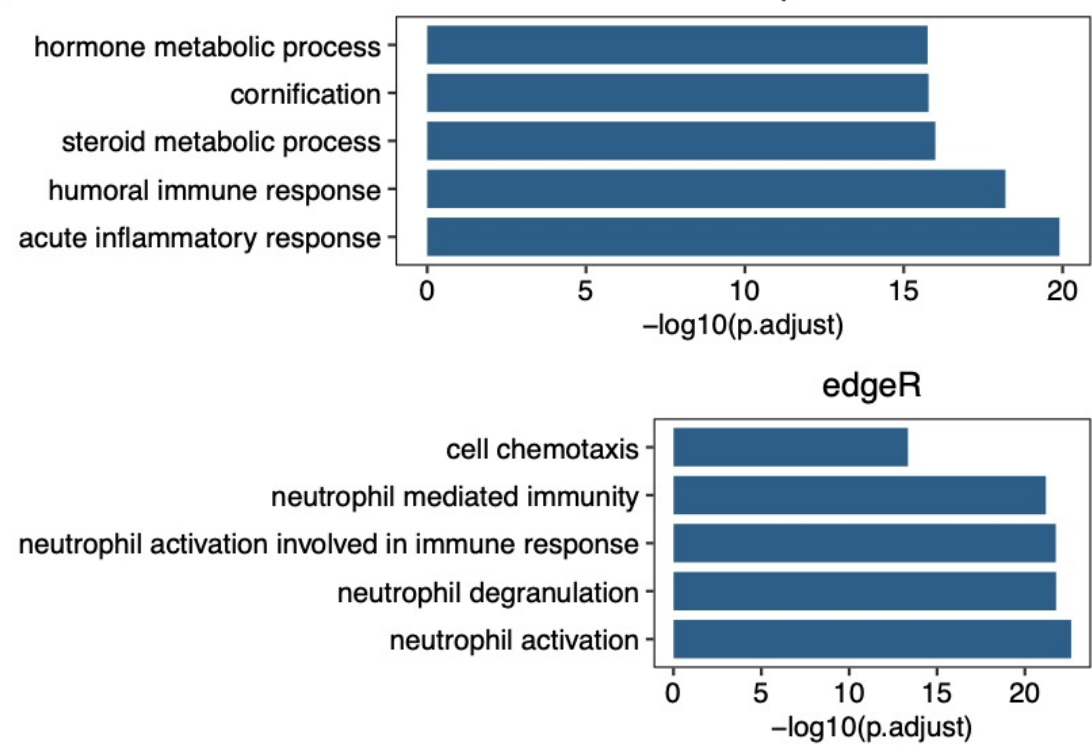

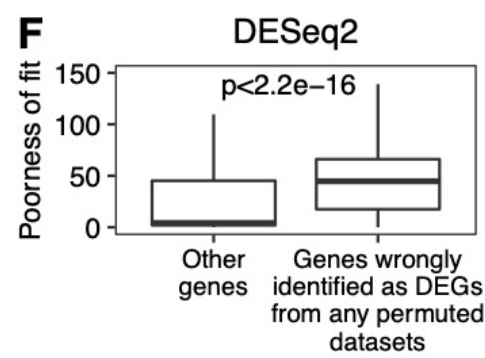

edgeR

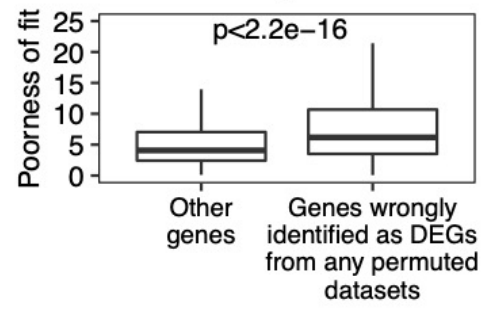




\section{Fig. 1. Exaggerated false DEGs identified by DESeq2 and edgeR from anti-PD-1 therapy}

\section{RNA-seq datasets.}

A. Barplot showing the average numbers of DEGs identified from 668 permuted datasets. The error bars represent the standard deviations of 668 permutations. The red dots indicate the numbers of DEGs identified from the original dataset.

B. The distributions of the number of permuted datasets where a gene was mistakenly identified as a DEG. The percentages corresponding to the numbers are listed in parentheses below the numbers.

C. Barplot showing the average numbers of DEGs identified from both the original dataset and the permuted datasets. The error bars represent the standard deviations of 668 permutations. The red dots indicate the numbers of DEGs identified from the original dataset.

D. Percentage of permuted datasets where a DEG identified from the original dataset was also identified as a DEG. The genes are sorted by absolute log2(fold-change) in the original dataset in decreasing order. The absolute $\log 2$ (fold-change) values corresponding to the ranks are listed in parentheses below the ranks. The line is fitted using the loess method, and the shaded areas represent $95 \%$ confidential intervals.

E. GO term enrichment for the DEGs identified from at least $10 \%$ permuted datasets. The top 5 enriched biological processes GO terms are shown. The analyses were performed using R package clusterProfiler. P.adjust represents the adjusted p-value using the Benjamini \& Hochberg method.

F. Boxplots showing the poorness of fitting the negative binomial model to the genes identified by DESeq2 or edgeR as DEGs from any permuted datasets vs. all the other genes. The poorness of fit for each gene is defined as its negative $\log _{10}(\mathrm{P}$-value $)$ from the goodness-of-fit test for the 
negative binomial distributions estimated by DESeq2 or edgeR. The p-value in each panel was calculated by the Wilcoxon rank-sum test to compare the two groups of genes' poorness-of-fit values.

A
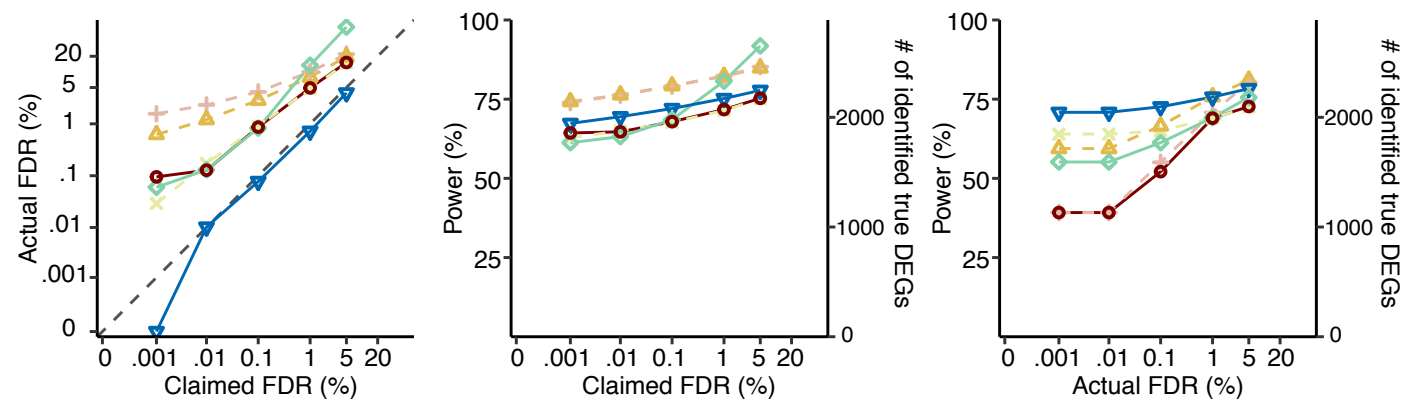

B
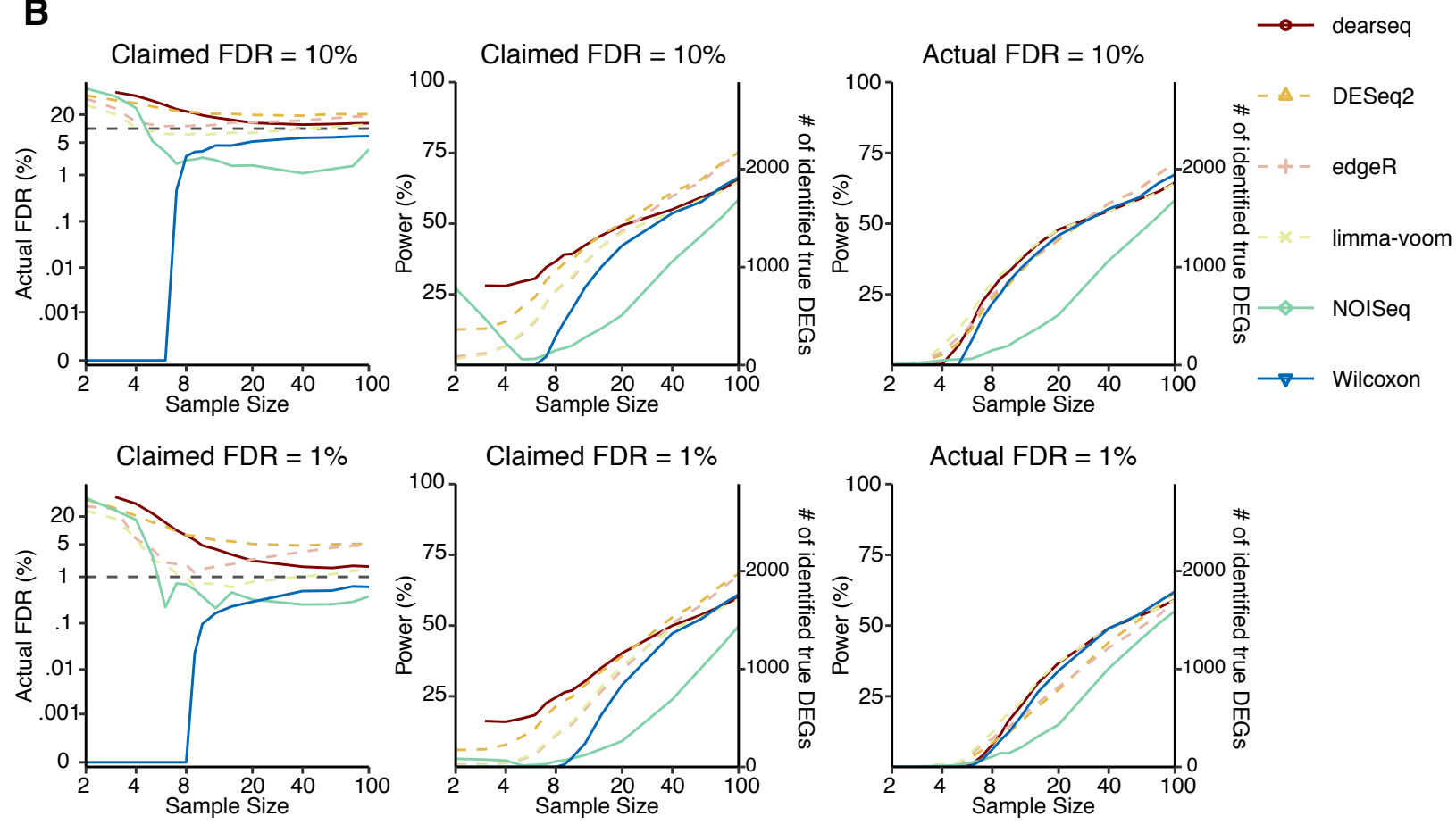

Fig. 2. Wilcoxon test has the best FDR control and power on heart left ventricle vs. atrial appendage GTEx datasets with synthetic ground truths.

A. The FDR control (left panel), power (middle panel) given the claimed FDRs, and power given the actual FDRs (right panel) under a range of FDR thresholds from $0.001 \%$ to $5 \%$. 
B. The FDR control (left), power given the claimed FDRs (middle), and power given the actual FDRs (right) for a range of per-condition sample sizes from 2 to 100, under FDR thresholds 10\% (top panels) and 1\% (bottom panels). The claimed FDRs, actual FDRs, and power were all calculated as the averages of 50 randomly down-sampled datasets. 